\title{
Critical illness neuro-myopathy (CINM) and focal amyotrophy in intensive care unit (ICU) patients with SARS-CoV-2: a case series
}

\author{
Nicola Alessandro Nasuelli ${ }^{1}$ (D) $\cdot$ Roberto Pettinaroli $^{1} \cdot$ Laura Godi $^{1} \cdot$ Claudio Savoini $^{2} \cdot$ Fabiola De Marchi $^{3}$. \\ Letizia Mazzini ${ }^{3} \cdot$ Federico Crimaldi $^{4} \cdot$ Aline Pagni $^{4} \cdot$ Cleta Patrizia Pompa ${ }^{4} \cdot$ Davide Colombo $^{4}$
}

Received: 14 July 2020 / Accepted: 10 October 2020 / Published online: 13 October 2020

(C) Fondazione Società Italiana di Neurologia 2020

\begin{abstract}
We found four patients with some characteristic phenotype in our ICU, characterized by focal hypotrophies of the shoulder girdle and the bilateral peroneal district and underlying critical illness neuro-myopathy. In our opinion, these hypotrophies are secondary to the prone position. Is our intention to start early treatment protocol with electrostimulation to evaluate the effectiveness in the prevention of critical illness and focal hypotrophies in ICU SARS-CoV-2 patients, to increase chances of returning to a preinfection functional status.
\end{abstract}

Keywords Sars-CoV-2 $\cdot$ COVID-19 $\cdot$ Critical illness neuro-myopathy $\cdot$ Intensive care unit $\cdot$ Pandemic

Coronavirus disease 2019 (COVID-19) is an infectious disease caused by a new strain of coronavirus, the severe acute respiratory syndrome coronavirus 2 (SARS-CoV-2). Within a few months of the first report in China, SARS-CoV-2 had spread worldwide, declared a global pandemic on 11 March 2020 by the World Health Organization. The outbreaks of COVID-19 have constituted a public health emergency of international concern. Respiratory distress is the most characteristic symptom, and more than half of patients with dyspnea need intensive care [1]. The estimated average time spent in intensive care unit (ICU) is described to be around 3 weeks. Prone position was recognized for its ability to improve oxygenation used as salvage therapy for refractory hypoxemia in ARDS, but potential complications could be facial pressure

Nicola Alessandro Nasuelli

nasuellinicola@gmail.com; nicolaalessandro.nasuelli@asl.novara.it

1 Department of Neurology, ASL NO, Borgomanero Hospital, Viale Zoppis 10, Borgomanero, 28021 Novara, Italy

2 Department of Physical Therapy, ASL NO, Borgomanero Hospital, Novara, Italy

3 Department of Neurology and ALS Centre, University of Piemonte Orientale, Maggiore della Carità Hospital, Novara, Italy

4 Intensive Care Department, ASL NO, Borgomanero Hospital, Novara, Italy ulcers, facial edema, lip trauma from endotracheal tube, and brachial plexus injury (due to arm extension) [2].

The aim of this communication is to highlight the relevance of neuromuscular involvement and its different clinical features in ICU patients with SARS-CoV-2. The neurotrophism of SARS-CoV-2 has been well demonstrated [3, 4]. Indeed, infected patients could show severe central nervous system involvement, such as necrotizing hemorrhagic encephalopathy, stroke, encephalitis, and epileptic seizures. Moreover, myalgia with elevated creatine kinase, hyposmia, and dysgeusia are well-known to be correlated at the infection $[5,6]$. In addition, some cases of acute peripheral nervous system disease $[7,8]$ are recently reported.

Neuromuscular disorders and critical illness neuromyopathy (CINM) could dramatically reduce the patient chances of returning to a preinfection functional status [8-10] and could have a detrimental role in respiratory failure outcome.

A 74-year-old Caucasian female (patient n. 1), non-smoker, with hypertension and hypercholesterolemia, in home therapy with sartanic and statins, was admitted in our hospital on 25 March 2020 for related COVID-19 respiratory failure. She early started noninvasive ventilation (NIV) in association with prophylactic heparin therapy (4.000 units/day), hidroxychloroquine $200 \mathrm{mg} /$ day, and antiretroviral drug. Ten days later, due to progressive worsening of the respiratory distress, she was admitted to ICU and started invasive ventilation with high peeps and pronation cycles $(16 \mathrm{~h}$ prone $/ 8$ 
Table 1 Nerve conduction study parameters

\begin{tabular}{llllll}
\hline Nerve & Side & Distal latency $(\mathrm{msec})$ & $\begin{array}{l}\text { Distal amplitude } \\
(\mathrm{mV})\end{array}$ & $\begin{array}{l}\text { Proximal amplitude } \\
(\mathrm{mV})\end{array}$ & $\begin{array}{l}\text { Velocity } \\
(\mathrm{m} / \mathrm{sec})\end{array}$ \\
\hline $\begin{array}{l}\text { Motor recordings } \\
\text { Common peroneal }\end{array}$ & $\begin{array}{l}\text { Right } \\
\text { Left }\end{array}$ & $\begin{array}{l}\text { Absent } \\
\text { Absent }\end{array}$ & $\begin{array}{l}\text { Absent } \\
\text { Tibial }\end{array}$ & Absent & \\
& Right & 3.1 & 3.4 & 3 & 41 \\
Ulnar & Left & 3.3 & 1.4 & 1.5 & 49.6 \\
Sensory recordings & Right & 2.3 & 1.1 & 1.3 & 60.8 \\
Sural & Right & Absent & & & \\
& Left & Absent & Absent & & \\
Superficial Peroneal & Right & Absent & Absent & & 48.7 \\
& Left & 1.5 & Absent & & 53.8 \\
\hline \multirow{2}{*}{ Ulnar } & Right & 1.6 & 8.7 & & \\
\hline
\end{tabular}

supine position). On the ninth day, tracheotomy was performed. After 3 days, the curarization was suspended, and a weaning was started by mechanical ventilation.

For bronchoaspirate positivity for A. baumannii and $S$. aureus and positive blood cultures for carbapenemresistant Klebsiella and C. albicans, she started antibiotic therapy. The patient always remains sleepy, asthenic, and unresponsive, despite the absence of sedation.

A neurological consultation was requested after 1 month in ICU. The examination showed drowsiness, no cranial nerve involvement, tetraplegia with diffuse hypotonia, and hypotrophy, mainly at shoulder girdle and to the peroneal district, without fasciculations. Deep tendon reflexes were absent with normal plantar response. There was no evidence of pyramidal and meningeal signs. Sensitivity was unevaluable due to mental status.

Laboratory results were clinically significant for lymphocytopenia: 0.71 (n.v $1.30-2.90 \mathrm{~m} / \mathrm{mmc}$ ), thrombocytopenia 64 (n.v 140-450 m/mmc), D-dimer 698 (n.v < 350 ng/ml), LDH 191 (n.v 120- U/l), PCR 6.32 ( n.v 0.00-0.50 $\mathrm{mg} / \mathrm{dl}$ ), and procalcitonin $3.89(\mathrm{n} . \mathrm{v}<0.5 \mathrm{ng} / \mathrm{mL})$. Cerebrospinal fluid showed 10 white blood cells and normal protein level; PCR for coronavirus-19 was negative. We also researched serum antibodies anti-GM1 IgG-IgM, anti-GQIb IgG-IgM, anti-GM2 IgG-IgM, anti-MAG, anti-sulfatide, antiGAD, and anti-CASPR2, all negative. The patient underwent electromyography (EMG): nerve conduction studies showed symmetrical axonal polyneuropathy, and needle study showed chronic neurogenic distal pattern with some proximal myopathic potential on deltoid muscle bilaterally (Table 1). The neurophysiological findings were compatible with CINM.

Due to muscular weakness, she needed continuous ventilation on pressure support (PSV) with gradually decreasing supports, until alternating cPAP and PSV cycles. She performed physiotherapy and cough machine therapy 4 times/ day. On 6 May, a worsening of the respiratory function requires to switch back to controlled ventilation. A progressive deterioration of the clinical conditions occurred (related to new superinfection), until death after 3 days.

We also studied three other SARS-CoV-2 patients (patient n. 2, 3, and 4) in ICU (Table 2, Fig. 1) who underwent invasive ventilation and pronation cycles due to respiratory distress. All these patients had similar phenotype, characterized by evident hypotrophy of the peroneal district and/ or of the shoulder girdle and underlying CINM at the EMG. The neurophysiological findings did not show nerve conduction blocks (particularly at the peroneal capitellum bilaterally).

Only one patient (patient n. 3), probably due to a younger age, had a positive outcome, even if characterized by slow recovery of motor skills, in particular due to foot flexion deficit, improved after intensive physiokinesitherapy.

Table 2 Features of patient 2, 3, and 4

\begin{tabular}{llllllll}
\hline Patient & Age & Gender & Phenotype* & EMG & Time in ICU & Superinfection & Outcome \\
\hline 2 & 71 years & M & U - L & CINM & $>3$ weeks & + & Deceased \\
3 & 60 years & M & U - L & CINM & $>3$ weeks & + & Alive \\
4 & 73 years & M & L & CINM & $>3$ weeks & - & Deceased \\
\hline
\end{tabular}

M, male; U, upper girdle hypotrophy; L, lower girdle hypotrophy; EMG, electromyography; CINM, critical illness neuro-myopathy; ICU, intensive care unit 
Fig. 1 Bilateral severe upper girdle hypotrophy (patient n. 2)

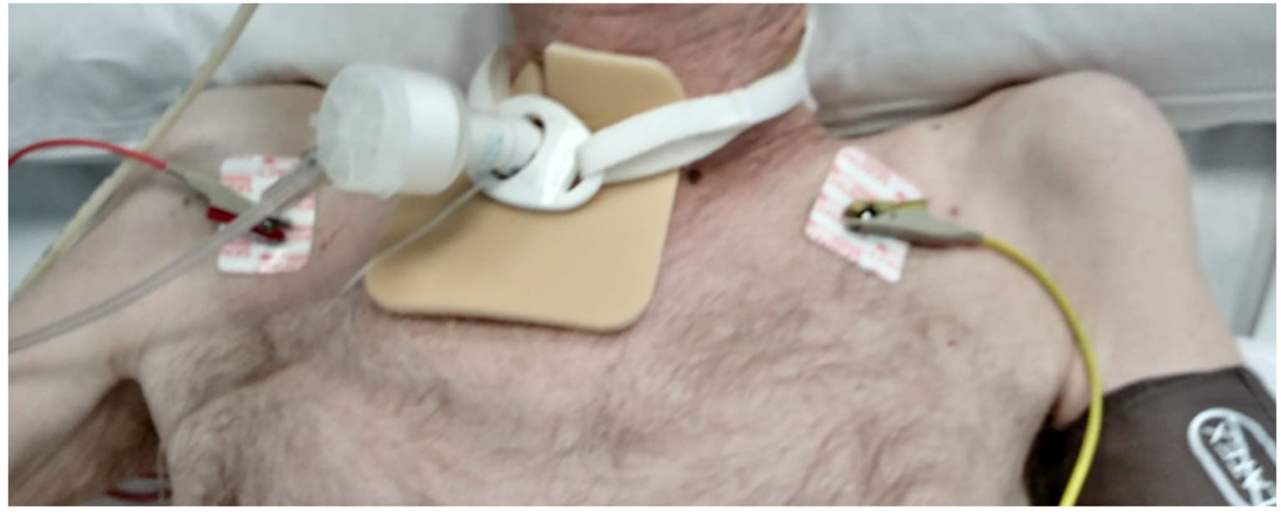

In conclusions, patients with SARS-CoV-2 infection in ICU are particularly predisposed to develop neuromuscular involvement for several modifiable and unmodifiable reasons, such as prolonged bed rest, intercurrent infections, and use of steroid therapy. In our opinion, these findings may be related to the prone position maintained for a long time by patients during the ventilation period and could be facilitated by underlying neuropathy, even further studies will be needed to demonstrate this evidence $[9,10]$.

It is our intention to start early treatment protocol with electrostimulation to evaluate the effectiveness in the prevention of CINM and focal hypotrophies in ICU SARS-CoV-2 patients, to increase chances of returning to a basal functional status.

Acknowledgments We would like to thank Marino Simona and Fantini Federica neurophysiopathology technicians for performing the exams.

\section{Compliance with ethical standards}

Conflict of interests The authors declare that they have no conflict of interest.

Ethics approval Observational case series; ethics approval not required.

\section{References}

1. Huang C, Wang Y, Li X, Ren L, Zhao J, Hu Y, Zhang L, Fan G, Xu J, Gu X, Cheng Z, Yu T, Xia J, Wei Y, Wu W, Xie X, Yin W, Li H, Liu M, Xiao Y, Gao H, Guo L, Xie J, Wang G, Jiang R, Gao Z, Jin Q, Wang J, Cao B (2020) Clinical features of patients infected with 2019 novel coronavirus in Wuhan, China. Lancet 395:497-506

2. Dalla Corte F, Mauri T, Spinelli E et al (2020) Dynamic bedside assessment of the physiologic effects of prone position in acute respiratory distress syndrome patients by electrical impedance tomography [published online ahead of print, 2020 May 22]. Minerva Anestesiol. https://doi.org/10.23736/S0375-9393.20.14130-0
3. Li Z, Liu T, Yang N, Han D, Mi X, Li Y, Liu K, Vuylsteke A, Xiang H, Guo X (2020) Neurological manifestations of patients with COVID-19: potential routes of SARS-CoV-2 neuroinvasion from the periphery to the brain [published online ahead of print, 2020 May 4]. Front Med, 1-9. doi:https://doi.org/10.1007/s11684020-0786-5

4. Li YC, Bai WZ, Hashikawa T (2020) The neuroinvasive potential of SARS-CoV2 may play a role in the respiratory failure of COVID-19 patients. J Med Virol 92(6):552-555. https://doi.org/ 10.1002/jmv.25728

5. Mao L, Jin H, Wang M, Hu Y, Chen S, He Q, Chang J, Hong C, Zhou Y, Wang D, Miao X, Li Y, Hu B (2020) Neurologic manifestations of hospitalized patients with coronavirus disease 2019 in Wuhan, China [published online ahead of print, 2020 Apr 10]. JAMA Neurol 77(6):1-9. https://doi.org/10.1001/jamaneurol. 2020.1127

6. Di Carlo DT, Montemurro N, Petrella G, Siciliano G, Ceravolo R, Perrini P (2020) Exploring the clinical association between neurological symptoms and COVID-19 pandemic outbreak: a systematic review of current literature. J Neurol 1-9. doi: https://doi.org/10. 1007/s00415-020-09978-y.

7. Toscano $\mathrm{G}$ et al (2020) Guillain-Barré syndrome associated with SARS-CoV-2. N Engl J Med 382(26):2574-2576. https://doi.org/ 10.1056/NEJMc2009191

8. Wang D, Hu B, Hu C, Zhu F, Liu X, Zhang J, Wang B, Xiang H, Cheng Z, Xiong Y, Zhao Y, Li Y, Wang X, Peng Z (2020) Clinical characteristics of 138 hospitalized patients with 2019 novel coronavirus-infected pneumonia in Wuhan, China. JAMA 323: 1061-1069. https://doi.org/10.1001/jama.2020.1585

9. Fan E (2012) Critical illness neuromyopathy and the role of physical therapy and rehabilitation in critically ill patients. Respir Care 57(6):933-946. https://doi.org/10.4187/respcare.01634

10. Brugliera L et al. (2020) J Rehabil Med 52: jrm00046 Journal Compilation (C) 2020 Foundation of Rehabilitation Information. ISSN 1650-1977 doi: https://doi.org/10.2340/16501977-2678 This is an open access article under the CC BY-NC license. www.medicaljournals.se/jrm REHABILITATION OF COVID-19 PATIENTS

Publisher's note Springer Nature remains neutral with regard to jurisdictional claims in published maps and institutional affiliations. 